\title{
LA IMAGEN DE LA MUJER POLÍTICA EN LOS MEDIOS DE COMUNICACIÓN
}

\author{
GLORIA GÓMEZ-ESCALONILLA, ANTONIO GARCÍA, MARINA SANTÍN, \\ RAQUEL RODRÍGUEZ Y JUAN TORREGROSA \\ Universidad Rey Juan Carlos (Madrid)
}

\section{INTRODUCCIÓN}

Este trabajo presenta las conclusiones de una investigación realizada sobre la representación de la mujer política en los medios de comunicación. Existen numerosas razones que explican la importancia de la presencia de la mujer en el ámbito político. El principal es que es el espacio del poder, un espacio especialmente dominado por el género masculino donde, además, han prevalecido y prevalecen los estereotipos de género más perjudiciales para la mujer. Este protagonismo casi indiscutible del varón ha propiciado que haya sido un escenario donde se han tomado medidas de discriminación positiva, como las cuotas o las listas paritarias, y sea por estas acciones o, con mayor probabilidad, por el mérito individual o el peso del progreso que reclama igualdad, también es la política un ámbito de cambio hacia una presencia cada vez más notable de líderes femeninas. Angela Merkel en la presidencia de Alemania, Michelle Bachelet en Chile, Cristina Kirchner en Argentina o Hillary Clinton en su carrera hacia la Casa Blanca ocupan cada vez más titulares en la información internacional. Y es que de la política interesa sobre todo su representación mediática porque el conocimiento que tiene la gente de este campo procede especialmente de los medios de comunicación, más que del conocimiento directo. Por ello esta investigación se centra particularmente en la representación mediática de la mujer política, entendiendo la trascendencia que tiene dicha representación para la configuración y construcción de la imagen de la mujer en la sociedad y como reflejo de lo que representa la mujer en el escenario político. 
De hecho existe una línea de investigación bastante asentada dentro de los estudios de género que aborda la imagen de la mujer a través de los medios ${ }^{1}$; la imagen de los políticos en la prensa ${ }^{2}$, y específicamente la imagen de la mujer política en los medios de comunicación ${ }^{3}$. La mayoría de estos estudios señalan que la característica básica de la representación mediática sobre la mujer política es la marginación con la que aparece y su papel casi de intrusa en la política, hasta el punto de que su presencia se «trivializa» al hacer referencia a su vida privada, sus planes domésticos o su estilo a la hora de vestir ${ }^{4}$. También han confirmado que, frente a los hombres, existen diferencias ya que, por ejemplo, se les dedica menos atención y son recurrentes las alusiones a la ropa, el peinado, o las relaciones familiares, es decir, todo lo que se supone típico de la feminidad y que pesa más que sus posiciones ideológicas o su experiencia política. De igual forma otros estudios ${ }^{5}$ revelan que los candidatos masculinos y femeninos se perciben teniendo rasgos y habilidades diferentes entre unos y otros: las mujeres son vistas como personas más compasivas, dignas de confianza y dispuestas al compromiso mientras que los hombres son vistos como más asertivos, agresivos y seguros de sí mismos.

1. Entre otros, BYerly, Carolyn M. and Ross, Karen: Women \& Media. A Critical Introduction, Maryland, Blackwell, 2006; LóPEz Díez, Pilar: Representación de género en los informativos de radio y televisión. Segundo Informe, Madrid, Instituto de la Mujer, RTVE, 2005; MUÑOZ, Blanca (coord.): Medios de comunicación, mujeres y cambio cultural, Madrid, Dirección General de la Mujer, 2001; Cunningham, George B.; SAGAS, Michael; Sartore, Melanie L.; Amsden, Michelle L.; SCHellHASE, Anne: "Gender representation in the NCAA news: is the glass half full or half empty? Sex Roles» A Journal of Research, June, 2004. Disponible en http://findarticles.com/p/articles/ mi_m2294/is_11-12_50?pnum=3\&opg=n6124159

2. Heldman, Caroline; Carroll, Susan J. and Olson, Stephanie: Gender Differences in Print Media Coverage of Presidential Candidates: Elizabeth Dole's Bid for the Republican Nomination, Washington, American Political Science Association, 2000. ADAY, Sean and DEVITT, James: Style over Substance. Newspaper Coverage of Female Candidates: Spotlight on Elizabeth Dole, Washington, National Press Club, 2000. Dow, Bonnie J. and ConDIT, Celeste M.: "The State of the Art in Feminist Scholarship in Communication", Journal of Communication, 55:3 (2005), pp. 448-478.

3. LEMISH, Dafna and DROB, Gili: "'All the time his wife': portrayals of first ladies in the Israeli press", Parliamentary Affairs, 55:1 (2002) pp. 129-142. PALMER, Barbara and SimON, Dennis M.: "When Women Run Against Women: The Hidden Influence of Female Incumbents in Elections to the U.S. House of Representatives, 1956-2002", Politics \& Gender, 1 (2005), pp. 39-63. BRADEN, Maria: Women politicians and the media, Lexington, KY, The University of Kentucky Press, 1996. AlEXANDER, Danrick and ANDERSEN, Karl Len: «Gender as a Factor in the Attribution of Leadership Traits. Political», Political Research Quarterly, 46:3 (1993), pp. 527-545. Ross, K.: «Sex, politics and the media: selling woman». Comunicación en Conferencia de IAMCR. Glasgow, Scotland. Julio 1998. Sanbonmatsu, Kira: "Gender Stereotypes and Vote Choice», American Journal of Political Science, 46:1 (2002), pp. 20-34. RuIz MOLINA, Encarna: «La imagen de las mujeres políticas en la prensa de información general». Jornadas para Reconstruir la Imagen Visual de las Mujeres en la España Contemporánea, Universidad Carlos III, 26-30 marzo 2001. Disponible en: http:// www.uc3m.es/uc3m/inst/MU/encarna_ruiz.html. URIARTE, Edurne: La incidencia de género en el reclutamiento de las elites políticas, Madrid, Instituto de la Mujer, 1995.

4. LEMISH, D. and DROB, G.: Op.cit.

5. Vid. los estudios de LeXANDER, Danrick and ANDERSEN, Karl Len: Op.cit., y SANBONMATSU, Kira: Op.cit. 
En línea con estos trabajos, el que aquí se presenta ${ }^{6}$ se basa en un análisis de contenido sobre una muestra de contenidos informativos (en los que se incluyen fotografías de prensa) representada por la sección de internacional, nacional y local de la prensa diaria así como sobre los informativos de televisión en la edición de máxima audiencia (tarde/noche). Los medios seleccionados han sido El País, ABC, El Mundo, TVE-1, Antena 3, Tele 5 y Telemadrid. El tiempo analizado ha sido de 30 días (del 20 de abril al 20 de mayo de 2006), y se ha tomado como unidad de análisis el político protagonista (hombre o mujer), registrando 3.212 unidades de información. En el trabajo de campo se han vaciado los datos que se han considerado de interés: variables de localización y de valoración, el nombre del político, su sexo, cargo, ámbito geográfico, partido político, tema de la noticia y variables relacionadas con la aparición del líder (acciones, atributos, vestuario, apariencia, escenario, compañía). Posteriormente se han categorizado los datos cualitativos para su tratamiento cuantitativo y se han procesado los datos obtenidos con un paquete estadístico para el establecimiento de frecuencias (absolutas y relativas) y cruces de contingencia. Las líneas que siguen resumen los resultados obtenidos.

\section{MUJERES Y HOMBRES EN POLÍTICA}

El estudio realizado confirma sin ningún género de dudas que los medios representan poco a la mujer política. El 83,5\% de las menciones corresponde a políticos varones mientras que el $16,5 \%$ corresponde a líderes de sexo femenino, prácticamente la misma cuota de participación de la mujer en política. Es decir, los medios representan la realidad existente, y como la realidad política es cosa de hombres, también en los medios se prioriza al género masculino.

Esta relación se reproduce además en todos y cada uno de los medios analizados pues representan más políticos varones que líderes mujeres, muchos más. El único medio que baja de los porcentajes superiores al $80 \%$ de presencia masculina es Telemadrid, que aporta un $22,3 \%$ de representación de cuota femenina en sus informativos, frente a un $77,7 \%$ de presencia masculina. Frente a Telemadrid, es Antena 3 el medio que menos mujeres representa, sólo mantiene un $9 \%$ frente a un $91 \%$ de presencia masculina.

Al cómputo de cuánto aparecen hombres y mujeres en los medios se añade también el análisis del lugar en el que aparecen o la jerarquía que presentan las informaciones. Teniendo en cuenta la clasificación que se ha propuesto en el estudio clasificando las noticias con máxima, media y mínima importancia, en cualquiera de las tres aparecen más los políticos varones. Pero no sólo aparecen más los varones porque hay más políticos varones; realizando análisis más exhaustivos, teniendo en cuenta la frecuencia relativa, se puede decir que

6. «La representación de la mujer en los medios de comunicación: comparación entre los contenidos y las percepciones de la mujer». Investigadores principales: Antonio García Jiménez y Gloria Gómez-Escalonilla. Equipo de investigación: Marina Santín Durán, Raquel Rodríguez y Juan Francisco Torregrosa (URJC). Proyecto financiado por la Dirección General de la Mujer de la Comunidad de Madrid y realizado en 2006. 
cuando aparece la mujer en los medios es más probable que se le otorgue una jerarquía normal que una jerarquía prioritaria, mientras que en los hombres se observa que aunque prima también la jerarquía media, es más probable que se les otorgue un trato preferencial. La diferencia de hombres y mujeres en la jerarquía superior supera el punto y medio, es decir, son los hombres los que más aparecen en las portadas y los que ocupan más espacio preferente en los medios de comunicación.

\subsection{La inexistente paridad en los partidos políticos}

La escasa referencia a la mujer política en los medios se debe, como se ha advertido, a la escasa presencia de la mujer en política, lo que supone que no funciona en la lógica mediática la aparición de los líderes en función del género, es decir, no por ser mujer, o por ser varón, los medios referencian a un político u otro. Más bien lo que funciona en las rutinas profesionales de los informadores es la aparición de los líderes en relación a sus respectivos partidos y en función de los rangos de poder que ocupan ellos y sus partidos. De este modo se ha podido comprobar que en la representación de los partidos políticos en los medios mantiene la lógica de representar a los partidos de acuerdo a su rango de poder. Los líderes que más aparecen son los líderes de los dos principales partidos en España: PP y PSOE. Aun siendo mayoritarios los políticos de los dos partidos PP y PSOE, también se pueden advertir diferencias significativas entre ellos, diferencias que benefician al partido socialista pues prácticamente la mitad de los políticos que se mencionan pertenecen al PSOE (49,8\%). Este protagonismo es hasta cierto punto esperable toda vez que es el partido que detenta el poder en el momento del estudio. El principal partido de la oposición, el PP, tiene una cuota de visibilidad mediática del $32,7 \%$. Los otros partidos que protagonizan la actualidad política, como Izquierda Unida, los nacionalistas catalanes (CIU y ERC) o vascos (PNV), tienen una representación minoritaria en relación a los grandes partidos. No aparecen apenas otros partidos cuya existencia en la vida política nacional es anecdótica.

Estos porcentajes minoritarios de los partidos políticos distintos a los dos mayoritarios se reducen todavía más cuando lo que se considera es la presencia femenina, pues cuando aparece una mujer política, lo normal y más habitual es que pertenezca al PP o al PSOE (92,8 \%). Solamente IU mantiene un escaso $3 \%$ de cuota femenina en los medios. También hay diferencias entre los dos partidos mayoritarios en lo que a presencia femenina se refiere, pues la relación favorece claramente al PSOE. La mitad de las mujeres políticas que aparecen en los medios pertenecen al partido socialista (50,4\%). Un 42,4\% de las mujeres que aparecen en los medios son del PP. A pesar de estos resultados, si se compara la cuota femenina con la masculina, en los dos partidos mayoritarios se comprueba que ambos quedan muy lejos de la paridad siendo mayoritaria la presencia de los hombres políticos en los dos. De todos los políticos del PP nombrados por los medios, el $75,2 \%$ son hombres mientras que el $24,8 \%$ son mujeres. De todos los políticos del PSOE, el 80,7 \% son hombres y el 19,3 \% mujeres. 
También existen diferencias estadísticamente significativas con respecto al partido político más representado según el medio de que se trate. Siempre, en todos los medios, los partidos más representados son PP y PSOE. En todos, excepto en Telemadrid, prima el PSOE. Esta relación advierte que los medios referencian ante todo los líderes de los partidos que gobiernan en su ámbito de cobertura. Todos los medios, excepto Telemadrid, tienen como ámbito de referencia el Estado español, como medios generalistas que son, donde gobierna el partido socialista. Es el medio regional el que no representa al PSOE sino al PP, que es el partido que gobierna en la Comunidad Autónoma que tiene de referencia. Estos datos traducen cierta lógica en el tratamiento mediático pues los medios siguen preferentemente la lógica de la jerarquía de poder (cargo) sobre el partido político y sobre todo sobre el género del líder que referencian.

Sin embargo, la comparación entre la proporción de menciones al PSOE o al PP del total de cada medio, datos que se reflejan en la tabla adjunta, advierte considerables diferencias. Como se puede ver, el que más referencia al PP es Telemadrid, seguido de Tele 5 y, a escasa distancia, $A B C$ y Antena 3 . Los que menos TVE-1 y en menor medida El Mundo y El País. Por lo que respecta al PSOE, los medios que se sitúan en el ranking de menciones son El Mundo y El País, manteniendo porcentajes similares, seguidos a escasa distancia por TVE-1. El que menos representa al partido socialista es Telemadrid, seguido de $A B C$ y Antena 3.

Tabla 1: Proporción de menciones a los partidos mayoritarios según los medios

\begin{tabular}{|l|c|c|c|c|c|c|c|}
\hline & ABC & Antena 3 & El Mundo & El País & Tele 5 & Telemadrid & TVE-1 \\
\hline PSOE & 47,8 & 48,6 & 57,5 & 57,5 & 46,3 & 39,9 & 56,6 \\
\hline PP & 31,3 & 32,4 & 28,6 & 30,5 & 33,3 & 51,2 & 22,9 \\
\hline N (100\%) & 1022 & 148 & 273 & 233 & 108 & 258 & 175 \\
\hline
\end{tabular}

\section{2. Ámbitos y tareas para hombres y para mujeres}

También jerarquía y poder representan los distintos escenarios o ámbitos en los que se puede desarrollar la política y el tipo de tareas asignadas, y en ambos casos se constata que los medios, posiblemente en consonancia con la realidad, relacionan los ámbitos y temas de mayor jerarquía con los políticos y los de menor importancia con las políticas, aunque siempre se advierte el predominio del género masculino en cualquier escenario y asunto relacionado con el poder.

Por lo que respecta al ámbito de referencia, del total de líderes analizados predominan los que se sitúan en el escenario nacional con el 36,5\% de los casos. También hay cierto protagonismo, y protagonismo equivalente, entre el ámbito autonómico e internacional con un porcentaje que ronda el $28 \%$. El ámbito que menos interesa a los medios es el local o municipal, pues los líderes que pertenecen a este ámbito representan un 6,5\%. En todos los ámbitos considerados, nacional, internacional, autonómico o local, el sexo de los políticos que prevalece es el masculino. No obstante, existe un ámbito de actuación 
donde las mujeres tienen cierto protagonismo en política, y ese es el local. En el ámbito local, el $34,1 \%$ de los líderes nombrados son mujeres.

En cuanto a las tareas abordadas en política, aunque son muchos los temas que toca la política y variados los problemas que trata, la política se centra sobre todo en sus asuntos, es decir, en decisiones que afectan a los partidos o a la gestión cotidiana del poder: elecciones, administración pública, nombramientos, pactos, relaciones entre políticos... También se tratan con cierta preferencia los temas de política exterior $(14,2 \%)$ o el terrorismo (13,2\%). Existen otros temas que aun siendo minoritarios en la agenda mediática cuentan con un porcentaje considerable de noticias. Son los de economía (5,2\%), infraestructura $(3,9 \%)$ o inmigración (3\%), cultura (3\%) y defensa (3\%). Frente a estos temas que conforman la actualidad, existen otros cuya presencia en los medios es anecdótica, no suponiendo ni un uno por ciento de las noticias analizadas, tal es el caso de la discriminación, educación, cuestiones de género, justicia, sanidad, trabajo o vivienda.

Es significativo destacar cómo siempre, en cualquier análisis realizado, resulta abrumadora la presencia de los hombres frente a las mujeres, excepto en algunos temas de trabajo que han resultado noticiosos para los medios analizados. Y es que está equilibrada la presencia de mujeres con respecto a hombres en cultura, en discriminación, en educación, medioambiente y sanidad, e incluso en el tema de género la presencia de la mujer supera a la presencia masculina, como si las cuestiones de género fueran cuestiones sólo de mujeres. En el resto de los temas: corrupción, defensa, economía, elecciones, infraestructuras, interior, justicia, política exterior, problemas administrativos y políticos, terrorismo, trabajo o vivienda predominan mayoritariamente los hombres. No es una casualidad esta distribución genérica, pues se advierte una nota común: es en la alta política donde predominan los hombres y es en las cuestiones sociales donde existe cierto lugar para la mujer política. Sólo en las cuestiones de género la presencia femenina supera la masculina. Esta distinción de temas responde a una cuestión claramente sexista, pues no hay diferencias significativas entre los dos partidos mayoritarios o el cargo ocupado en los temas que protagonizan.

Estos datos se relacionan con las conclusiones obtenidas por Sanbonmatsu que señala diferentes competencias asignadas a hombres y mujeres ${ }^{7}$. Según estos estudios se prefiere a mujeres en materias vinculadas a la educación, la salud, los derechos, el medioambiente y la asistencia social y los hombres estarían más preparados para tratar con los impuestos y presupuestos, la resolución de crímenes, la defensa nacional y la política exterior.

\subsection{La apariencia de políticos y políticas en los medios}

Como cabría esperar, los políticos, ya sean hombres o mujeres, optan en su mayoría por una apariencia formal y seria, al menos cuando aparecen reflejados en los medios de comunicación. Efectivamente en un $84,3 \%$ de los casos apare-

7. SANBOMATSU, Kira: Op.cit. 
cen con una apariencia formal. Tan sólo en un 15,7\% de las ocasiones aparecen vestidos de forma informal. Aunque esta es la pauta general e indiscutible, que funciona sin diferencias en razón del partido político, sí se observan diferencias, de cinco puntos, en razón del género, y es que las mujeres eligen un estilo formal con más frecuencia que los hombres (un $88,2 \%$ las mujeres frente a un $83,6 \%$ los hombres) y en menos ocasiones (un $11,8 \%$ las mujeres, un $17,4 \%$ los hombres) optan por el estilo informal.

Otra variable importante en la consideración de la imagen de los políticos en los medios es la compañía, pues en el $65 \%$ de los casos analizados suelen aparecer acompañados de otros personajes, tanto mujeres como varones, aunque es más probable que si aparece sin compañía, el líder político sea una mujer. Ahora bien, cuando se trata de analizar de quién se acompañan, de nuevo se constata una presencia mayoritariamente masculina. Tanto los políticos varones como las mujeres políticas suelen aparecer rodeados de varones, aunque no en la misma proporción, es decir, cuando aparece una compañía mayoritariamente femenina es porque el líder referenciado también es mujer.

Otro aspecto a considerar en el análisis de la apariencia de los políticos es el escenario donde aparecen. Pues bien, los escenarios en los que se mueven los políticos, por lo menos lo que nos ofrecen los medios, son bastante recurrentes, pues la mitad de las veces el escenario se corresponde con una sala de prensa, es decir, en las comparecencias públicas ante los medios, u otras salas de reuniones. Cuando no aparecen en una sala de prensa, lo hacen en la calle (21\%). En muy pocas ocasiones aparece en su despacho $(2,6 \%)$ o en un escenario privado $(1,8 \%)$. Y también en este aspecto existen diferencias, aunque no ciertamente muy notables, en los escenarios que rodean a los hombres políticos frente a los escenarios que rodean a las mujeres políticas, pues aun siendo el escenario más frecuente la sala de prensa y el que menos la calle entre hombres y mujeres, no lo es en la misma proporción, pues los hombres ganan en las salas de prensa y la mujer en la calle, ofreciendo una imagen más institucional el líder masculino y más cercana, más accesible y más «normal» la política mujer.

\section{LÍDERES POLÍTICOS CON NOMBRE Y APELLIDO}

Del total del periodo analizado, han resultado un total de 3.212 menciones a unos 600 políticos o políticas. Esta relación advierte que los actores de la política, o al menos los que aparecen en los medios, son numéricamente considerables y cuanto menos variados y heterogéneos. Al mismo tiempo hay que advertir que algunos políticos o políticas aparecen en escasas ocasiones pues la mayoría sólo resultan mencionados una, dos, tres o cinco veces. Los protagonistas de la política en los medios, consignando los que aparecen más de 10 veces en el periodo considerado, son 54 personajes. Pues bien, la mayoría de esos personajes son masculinos. De los 54 líderes, sólo 10 son mujeres, y 44 son hombres, es decir, la relación es de cerca del $80 \%$ de cuota masculina frente a un escaso $20 \%$ femenino. Si bien estos datos son los que resultan de la consideración de los líderes con más de 10 menciones, existen lógicamente diferencias entre la frecuencia de aparición entre ellos. Existen personajes que 
superan las cien apariciones, e incluso las doscientas. Por orden de aparición, la relación es la que sigue:

\begin{tabular}{|l|l|}
\hline José Luis Rodríguez Zapatero & 208 \\
\hline Mariano Rajoy & 152 \\
\hline Pasqual Maragall & 124 \\
\hline Esperanza Aguirre & 101 \\
\hline Evo Morales & 105 \\
\hline
\end{tabular}

Como se puede ver en la tabla de los cinco líderes más nombrados sólo una persona es de sexo femenino, aunque esta diferencia responde más bien a la correspondencia de género con respecto al cargo. Aparece en primer lugar el presidente del gobierno, José Luis Rodríguez Zapatero, y en segundo lugar el líder de la oposición, Mariano Rajoy. Son los dos principales rostros de la política española, los rostros de los dos partidos principales, los líderes del PSOE y del PP, ambos varones. Siguen en esta relación los entonces presidentes de las principales comunidades autónomas del país: de Cataluña, Pascual Maragall, y de Madrid, Esperanza Aguirre. Este par aparece enfrentado pero no tanto por el género, ni siquiera por el partido político, aparece enfrentado por la situación geográfica que representan. La inclusión de Evo Morales, el presidente de Bolivia, se debe a la agenda, pues el periodo de estudio coincidió con el anuncio de la nacionalización de los pozos bolivianos, aspecto que afecta a la compañía española Repsol.

Es interesante también destacar que existen diferencias significativas estadísticamente hablando entre la aparición de los líderes y los diferentes medios de comunicación analizados, pues no en todos los medios prima la mención al Presidente del Gobierno. Y es que tanto en Telemadrid como en Antena 3 no es Rodríguez Zapatero el líder más nombrado. En la cadena autonómica de Madrid, como puede resultar esperable, la protagonista indiscutible es Esperanza Aguirre, la presidenta de la Comunidad, mientras que en la cadena Antena 3 el líder político que resulta más mencionado es Mariano Rajoy.

Tabla 2: Proporción de menciones a los principales líderes según los medios

\begin{tabular}{|l|c|c|c|c|c|c|c|}
\hline & ABC & Antena 3 & El Mundo & El País & Tele 5 & Telemadrid & TVE-1 \\
\hline Zapatero & 5,3 & 7,3 & 8,7 & 6,9 & 6,4 & 5,8 & 8,6 \\
\hline Rajoy & 3,2 & 9,7 & 4,8 & 4,6 & 5,7 & 6,8 & 6,1 \\
\hline N (100\%) & 1422 & 206 & 413 & 479 & 141 & 309 & 244 \\
\hline
\end{tabular}

También resulta interesante contrastar las diferencias entre los líderes autonómicos más nombrados según los medios, pues también son significativas pues todos los medios, excepto Telemadrid, citan más al presidente catalán que a la presidenta madrileña. Ello no implica necesariamente un criterio sexista, porque también corresponden a partidos distintos y a posturas políticas bien diferenciadas, aunque también puede explicarse porque la actualidad de la agenda de esos días priorizase la comunidad catalana sobre la de Madrid. Sea por lo que fuere lo cierto es que la única mujer que tiene oportunidad de aparecer en 
La imagen de la mujer política en los medios de comunicación

la ventana mediática aparece mucho menos que el que se podría considerar su homólogo varón.

Tabla 3: Proporción de menciones a los principales líderes regionales según los medios

\begin{tabular}{|l|c|c|c|c|c|c|c|}
\hline & ABC & Antena 3 & El Mundo & El País & Tele 5 & Telemadrid & TVE-1 \\
\hline Maragall & 3,0 & 3,4 & 7,0 & 4,0 & 3,5 & 3,6 & 3,7 \\
\hline Aguirre & 2,9 & 0,5 & 2,7 & 3,1 & 2,8 & 11,7 & 1,2 \\
\hline $\mathbf{N}(\mathbf{1 0 0} \%)$ & 1422 & 206 & 413 & 479 & 141 & 309 & 244 \\
\hline
\end{tabular}

Aunque con menos de frecuencia de aparición, también hay que considerar otros líderes que aparecen con cierto protagonismo en los medios de comunicación, con menciones que superan los 50 casos:

\begin{tabular}{|l|c|}
\hline María Teresa Fernández de la Vega & 85 \\
\hline George Bush & 74 \\
\hline José Antonio Alonso & 72 \\
\hline Alberto Ruiz Gallardón & 70 \\
\hline Josep Lluis Carod Rovira & 65 \\
\hline Hugo Chávez & 63 \\
\hline Alfredo Pérez Rubalcaba & 60 \\
\hline
\end{tabular}

Como se puede comprobar, encabeza la lista una mujer, la Vicepresidenta del Gobierno, que se acompaña en este ranking de dos ministros, varones los dos, el ministro de Interior y el de Defensa. También interesa destacar que en la lista de políticos con nombre y apellidos está Alberto Ruiz Gallardón, el único alcalde que aparece con más de 50 apariciones. No en vano es el alcalde de la capital, Madrid. Su presencia en los diferentes medios también arroja diferencias notables, aunque no se pueden hablar de diferencias significativas, salvo en Antena 3, que no ofrece ninguna referencia al alcalde madrileño en estas fechas. Por lo demás cabe decir que es Telemadrid, como puede resultar lógico, el medio que más le menciona en proporción a los líderes que refleja en sus informativos, seguido de El País y $A B C$. Frente al alcalde de Madrid, del Partido Popular, hay que mencionar a otro líder catalán, no ya el alcalde de Barcelona, sin apenas presencia en los medios en esta época, sino el presidente de Esquerra Republicana Josep Lluis Carod Rovira. Curiosamente los medios que más reflejan a este líder son los que menos reflejaban a Gallardón: Tele 5, Antena 3 y El Mundo. Por lo que respecta a los otros líderes, son todos ellos del ámbito internacional, y destaca el Presidente de Estados Unidos y el Presidente de Venezuela que, junto a Evo Morales que aparece en el ranking preferencial, son los tres protagonistas indiscutibles de las páginas de internacional en esas fechas.

De estos políticos con nombre propio, nombre ganado por el cargo, por el país, por personalidad o por la polémica que conllevan, hay que destacar que predominan los hombres, como puede resultar hasta cierto punto lógico debido a la trayectoria histórica seguida en este mundo político dominado incluso en nuestros días por el sexo masculino. Por eso es de destacar el papel de dos mujeres que han abierto camino en este mundo de hombres y merecen, también 
en la ventana mediática, un papel protagonista de la actualidad política. Esas dos mujeres que destacan en nuestro país son Esperanza Aguirre y María Teresa Fernández de la Vega. Es de destacar que el cargo que ostentan no es comparable en ningún caso, pues Fernández de la Vega se sitúa a un nivel superior en el escalafón político aunque sin tanto protagonismo en las páginas de los medios. Es de destacar también que las dos mujeres enfrentan dos opciones políticas que se presentan en el escenario español como los dos partidos mayoritarios: PP y PSOE.

Mucho más interesante resulta analizar la relación del personaje con el tono o juicio de la información. A pesar de las escasas frecuencias encontradas, y de la cautela con que debemos interpretar estos datos, toda vez que el tono se puede referir no sólo al líder político sino también al hecho noticioso que se trata de contar, se han encontrado relaciones estadísticamente significativas entre el personaje y el tono de la información, lo cual supone que los medios no consideran a todos los políticos igual y que dependiendo del líder de que se trate la información tenderá a ser más positiva, más negativa o más neutral:

Tabla 4: Proporción de menciones negativas, positivas o neutras según los líderes

\begin{tabular}{|l|r|r|r|c|}
\hline & Negativo & Neutro & Positivo & (N) $\mathbf{1 0 0 \%}$ \\
\hline José Luis Rodríguez Zapatero & 27,8 & 65,8 & 6,2 & $(208)$ \\
\hline Mariano Rajoy & 14 & 57 & 29 & $(152)$ \\
\hline Pasqual Maragall & 31 & 65 & 4 & $(124)$ \\
\hline Esperanza Aguirre & 6,4 & 47,7 & 45,8 & $(101)$ \\
\hline Evo Morales & 26,9 & 71,1 & 1,9 & $(105)$ \\
\hline$M^{a}$ Teresa Fdez. de la Vega & 38 & 58,3 & 3,5 & $(85)$ \\
\hline George Bush & 6,7 & 89,1 & 4 & $(74)$ \\
\hline José Antonio Alonso & 20 & 77,1 & 2,8 & $(72)$ \\
\hline Alberto Ruiz Gallardón & 2,9 & 64,7 & 32,3 & $(70)$ \\
\hline Joseph Lluis Carod Rovira & 33,3 & 65 & 1,5 & $(65)$ \\
\hline Hugo Chávez & 26,2 & 73,7 & 0 & $(63)$ \\
\hline Alfredo Pérez Rubalcaba & 38,3 & 53,3 & 8,3 & $(60)$ \\
\hline
\end{tabular}

Como se puede comprobar en la tabla, prima sobre todo la valoración neutra o la ausencia de valoración, en correspondencia con la objetividad o al menos imparcialidad de la información periodística. En prácticamente todos los líderes importantes son más de la mitad las informaciones en las que la información es neutral por lo que priman las informaciones imparciales sobre las parciales, excepto en el caso de Esperanza Aguirre. Es la única persona sobre la que los medios tienden a valorar más, ya que en ese caso las informaciones con valoración superan las imparciales. Con respecto a los líderes nacionales, y atendiendo al signo de la valoración, se observa una pauta que se muestra de manera rotunda: es más probable que el signo sea negativo si el líder político es del PSOE que del PP. Es decir, los políticos populares superan las noticias positivas a las negativas. Los políticos socialistas superan las noticias negativas a las positivas. Los medios tratan muchísimo mejor a los líderes del PP que a los líderes del PSOE, 
y esa pauta no respeta cargos ni respeta géneros. Efectivamente tanto en el caso del Presidente del Gobierno como en el caso de la Vicepresidenta se superan las noticias negativas a las positivas, con 21,5 puntos de diferencia en el caso de Zapatero y 34,5 puntos de diferencia con respecto a la Vicepresidenta. Son diferencias significativamente altas y significativamente desfavorables para los altos mandatarios españoles. Contrasta además con el tratamiento que reciben tanto el Presidente del PP, el líder de la oposición Mariano Rajoy como con el tratamiento a Esperanza Aguirre. En ambos casos superan las noticias positivas a las negativas, con una diferencia porcentual de 15 puntos en el caso de Rajoy y casi 40 puntos en el caso de la presidenta de la Comunidad de Madrid.

Los ministros, en este caso José Antonio Alonso y Alfredo Pérez Rubalcaba, también superan la valoración negativa sobre la positiva, con diferencias de veinte y treinta puntos respectivamente. Mientras, el Alcalde de Madrid, Alberto Ruiz Gallardón, del Partido Popular, protagoniza más noticias positivas que negativas, un $30 \%$ más. Los medios son también muy críticos son respecto a los líderes catalanes, ya que tanto en el caso de Pascual Maragall como en el de Josep Lluis Carod Rovira, las diferencias entre las informaciones positivas y negativas son muy notables, a favor de las negativas.

Así pues, los medios en su tratamiento informativo dejan a un lado la profesionalidad que exigiría una imparcialidad política; dejan también a un lado la labor de salvaguarda del poder político propia de este cuarto poder que exigiría ser crítico con los políticos que ejercen el poder, también en el ámbito autonómico o municipal, y se decantan por una lógica ideológica y partidista, con matices de cierto centralismo madrileño. En este sentido podemos concluir que no hay estereotipos de género más allá de los que imperan en el propio ámbito político, y que el tratamiento favorable o desfavorable responde al partido político y no al género del líder en cuestión. Los medios en este sentido no son sexistas, son claramente partidistas.

\section{CONCLUSIONES}

De todo lo dicho, y a modo de conclusión, se puede decir que los medios representan poco a la mujer política. De cada seis líderes que aparecen en los medios de comunicación, cinco son varones y sólo una es mujer. Ahora bien, esta relación remite a una realidad tan rotunda como los datos, y es que la mujer no está en las páginas de los medios porque tampoco está en la actualidad de la política. Los medios reflejan un bipartidismo que realmente polariza la política reproduciendo las cuotas genéricas establecidas por esos mismos partidos, y la visibilidad de las mujeres en los medios es la misma visibilidad que tienen en las instituciones y en los cargos que ostentan. Los medios, a la postre, sólo hacen que reproducir un mundo tremendamente injusto con el género femenino.

A ello hay que añadir el hecho de que los medios no funcionan tanto respondiendo a prejuicios de género cuanto a prejuicios de partido, es decir, en la valoración, y no tanto en la frecuencia de aparición, se privilegian a los líderes del Partido Popular sobre los líderes del PSOE o de otros partidos, sobre todo nacionalistas. Es decir, a pesar de que a los medios se les puede y debe exigir 
neutralidad o imparcialidad informativa, se decantan por una determinada ideología, y coincide esa ideología con los intereses del Partido Popular. Esa preferencia por los líderes populares se constata de forma independiente del cargo que ocupan los líderes, del ámbito de actuación y, sobre todo, del sexo que tengan.

Ahora bien, una lectura más exhaustiva de los datos, analizando los árboles y no tanto el bosque, advierte de algunas lógicas no esperables, menos profesionales y también perjudiciales de la representación de la mujer en los medios de comunicación:

En primer lugar porque aunque ciertamente exista discriminación hacia la mujer en la vida política, existen algunos espacios donde, políticamente, se ha querido representar la paridad: en el gabinete ministerial y en la presidencia de la nación. Pues bien, en los medios no existe tal paridad, ni en la representación de los ministros ni en la representación del gobierno de España, pues aparecen mucho más los ministros que las ministras, y también mucho más el Presidente que la Vicepresidenta del Gobierno, a pesar de que en las comparecencias con la prensa se priorice la presencia de Fernández de la Vega.

En segundo lugar porque cuando se representa a la mujer política, se la relaciona con los temas menos importantes de la agenda política. Cierto que en la realidad nacional no existe esa equivalencia entre hombres y mujeres, y ni siquiera en el gabinete ministerial, único reducto político donde existe paridad real, se respeta ese equilibrio pues las mujeres ministras ostentan las carteras menos importantes, pero los medios no sólo reproducen esta realidad sino que agudizan esa lógica de situar a los líderes masculinos como los protagonistas de la alta política y reservar a las líderes femeninas en ámbitos temáticos relacionados con la sociedad y la cultura.

$Y$ en tercer lugar porque los resultados del estudio referidos a la jerarquía también priorizan, en datos absolutos y relativos, a los hombres sobre las mujeres políticas. No sólo aparecen poco en los medios, sino que su aparición no se destaca de entre otros contenidos mediáticos, no aparecen tanto en la portada, y las informaciones que protagonizan no suelen ocupar un lugar preferente en el orden de aparición ni en tiempo o espacio del medio en cuestión.

En definitiva, la mujer política apenas tiene visibilidad mediática, porque tampoco tiene protagonismo político. Las mujeres que aparecen, además, aparecen con nombre y apellido, y lo hacen en relación a su cargo y al partido que representan. Sobresale en este sentido la Presidenta de la Comunidad de Madrid, no solo por ser la mujer que más presencia tiene en los medios sino también por el tratamiento favorable que ostenta en el tratamiento mediático, pues no solamente es la mujer mejor tratada sino la líder mejor considerada por los medios de comunicación, aunque no se pueda concluir de ello que es por el hecho de ser mujer. Sea por lo que fuere, el hecho constatable es que en el panorama de la información política, el sexo femenino tiene realmente pocos rostros. Uno es el de la vicepresidenta del Gobierno $\mathrm{M}^{a}$ Teresa Fernández de la Vega, y el otro la Presidenta de la Comunidad, Esperanza Aguirre, que han logrado hacerse un hueco en la ventana mediática, difícil cometido en la vida 
política, un ámbito que sigue siendo en pleno siglo XXI un reducto dominado casi exclusivamente por el sexo masculino.

\section{BIBLIOGRAFÍA}

ADAY, Sean and DEVITT, James: Style over Substance. Newspaper Coverage of Female Candidates: Spotlight on Elizabeth Dole, Washington, National Press Club, 2000.

AleXANDER, Danrick and ANDERSEN, Karl Len: "Gender as a Factor in the Attribution of Leadership Trait», Political Research Quarterly, 46:3 (1993), pp. 527-545.

Byerly Carolyn M. and Ross, Karen: Women \& Media. A Critical Introduction, Maryland, Blackwell, 2006.

BRADEN, Maria: Women politicians and the media, Lexington, KY, The University of Kentucky Press, 1996.

Cunningham, George B.; Sagas, Michael; Sartore, Melanie L.; AmSDen, Michelle L.; SCHELlHASE, Anne: "Gender representation in the NCAA news: is the glass half full or half empty? Sex Roles», A Journal of Research, June, 2004. Disponible en http:// findarticles.com/p/articles/mi_m2294/is_11-12_50?pnum=3\&opg=n6124159

Dow, Bonnie J. and CondIT, Celeste M.: «The State of the Art in Feminist Scholarship in Communication", Journal of Communication, 55:3 (2005), pp. 448-478.

FOUNTAINE, Susan and MCGREGOR, Judy: «Reconstructing gender for the 21st century: News media framing of political», en Power, M. R. (ed.): ANZCA 2002, Australian \& New Zealand Communication Association, 23rd Annual Conference, July 10-12, Coolangatta, Queenslan, 2002. Disponible en: http://www.bond.edu.au/hss/communication/ANZCA/papers/JMcGregorSFountainePaper.pdf

Heldman, Caroline; CARROlL, Susan J. and Olson, Stephanie: Gender Differences in Print Media Coverage of Presidential Candidates: Elizabeth Dole's Bid for the Republican Nomination, Washington, American Political Science Association, 2000.

LEMISH, Dafna and DROB, Gili: "'All the time his wife': portrayals of first ladies in the Israeli press», Parliamentary Affairs, 55:1 (2002), pp. 129-142.

LÓPEZ DíEZ, Pilar: Representación de género en los informativos de radio y televisión. Segundo Informe, Madrid, Instituto de la Mujer; RTVE, 2005. Disponible en: http://www.rtve. es/oficial/iortv/SegundoInforme.pdf

MUÑOZ, Blanca (coord).: Medios de comunicación, mujeres y cambio cultural, Madrid, Dirección General de la Mujer, 2001.

PALMER, Barbara and SiMON, Dennis M.: «When Women Run Against Women: The Hidden Influence of Female Incumbents in Elections to the U.S. House of Representatives, 1956-2002», Politics \& Gender, 1 (2005), pp. 39-63.

Ross, K. «Sex, politics and the media: selling woman». Comunicación en Conferencia de IAMCR. Glasgow, Scotland. Julio 1998.

RUIZ MOLINA, Encarna: "La imagen de las mujeres políticas en la prensa de información general». Jornadas para Reconstruir la Imagen Visual de las Mujeres en la España Contemporánea, Universidad Carlos III, 26-30 marzo 2001. Disponible en: http://www.uc3m.es/uc3m/inst/MU/encarna_ruiz.html

SANBONMATSU, Kira: "Gender Stereotypes and Vote Choice», American Journal of Political Science, 46:1 (2002), pp. 20-34.

URIARTE, Edurne. La incidencia de género en el reclutamiento de las elites políticas, Madrid, Instituto de la Mujer, 1995. 